In the absence of definite information about the problem to be considered, it is difficult to obtain an estimate of the total number of iterations required for the last five methods.

For our method, we obtain:

I. Elimination

$$
\text { Auxiliary Method }
$$

II. Seidel (one iteration)

III. Relaxation (one iteration)

IV. Gradient method (one iteration)

V. Conjugate gradient method, symmetric positive definite matrix (one iteration)

VI. Conjugate gradient method, general matrix (one iteration)

$\begin{array}{cc}\text { Multiplications } & \text { Additions } \\ n^{4} / 12 & n^{4} / 12 \\ n^{3} / 3 & n^{3} / 3 \\ n^{3} / 3 & n^{3} / 3 \\ 2 n^{3} / 3 & 2 n^{3} / 3 \\ & \\ n^{3} / 3 & n^{3} / 3 \\ n^{3} & n^{3}\end{array}$

3.6. Numerical Comparison. For a $10 \times 10$ determinant, we obtain the following estimates for the number of operations required for the methods discussed:

\section{Method}

1. Expansion in elements

2. Expansion in minors

3. Chio's method

4. Tridiagonal method

5. Auxiliary method (by means of solution of linear equations) I

II (one iteration)

III (one iteration)

IV (one iteration)

$\mathrm{V}$ (one iteration)

VI (one iteration)

\section{Multiplications}

$3.26 \times 10^{7}$

$6.23 \times 10^{6}$

330

1300

1000

330

330

660

330

1000
Additions

$3.63 \times 10^{6}$

330

660

1000

330

330

660

330

1000

John Jay Hopkins Laboratory for Pure and Applied Science General Atomic Division of General Dynamics Corporation San Diego, California

1. K. S. Kuntz, Numerical Analysis, McGraw-Hill, New York, 1957.

2. E. M. GRABBE, S. RAMO \& D. E. WooldRIDGE, Handbook of Automation, Computation and Control, v. I, John Wiley and Sons, New York, 1958.

\title{
Coefficients in Quadrature Formulas
}

\section{By A. H. Stroud}

The following result is well known (see, for example, Krylov [2], p. 104, or Szego [3], p. 48):

THEOREм 1. If $w(x)$ is nomnegative throughout the finite or infinite segment $[a, b]$ and if the quadrature formula

$$
\int_{a}^{b} w(x) f(x) d x \simeq \sum_{i=1}^{n} A_{i} f\left(x_{i}\right)
$$

Received October 29, 1962. 
is exact for all polynomials of degree $\leqq 2 n-2$ then the coefficients $A_{i}(i=1,2, \cdots, n)$ are all positive.

Here the $x_{i}$ are assumed to be real numbers. The usual method for proving this theorem is to obtain the following representation for the $A_{i}$ :

$$
A_{i}=\int_{a}^{b} w(x)\left[\frac{\omega(x)}{\left(x-x_{i}\right) \omega^{\prime}\left(x_{i}\right)}\right]^{2} d x \quad(i=1,2, \cdots, n)
$$

where $\omega(x)=\left(x-x_{1}\right)\left(x-x_{2}\right) \cdots\left(x-x_{n}\right)$. If $w(x)$ changes sign then this representation still holds, but in general we are not able to determine from it how many of the $A_{i}$ are positive and how many negative.

In Theorem 2 below we give a method for determining the number of positive and negative $A_{i}$ in (1) for a more general weight function.

In the remainder of this note we assume that $w(x)$ is any function for which the moments

$$
c_{i}=\int_{a}^{b} w(x) x^{i} d x \quad(i=0,1,2, \cdots)
$$

are defined and finite and for which the moment matrix

$$
C_{n}=\left[\begin{array}{cccc}
c_{0} & c_{1} & \cdots & c_{n-1} \\
c_{1} & c_{2} & \cdots & c_{n} \\
\vdots & \vdots & \vdots & \vdots \\
c_{n-1} & c_{n} & \cdots & c_{2 n-2}
\end{array}\right]
$$

is nonsingular.

Theorem 2. If $C_{n}$ is congruent (in the terminology of Gantmacher [1], p. 296) to a diagonal matrix with $p$ positive and $q$ negative diagonal elements $(p+q=n)$-or equivalently, if $C_{n}$ has $p$ positive and $q$ negative eigenvalues-then a quadrature formula (1) which is exact for all polynomials of degree $\leqq 2 n-2$ has $p$ positive and $q$ negative coefficients.

Proof. The condition that (1) be exact for all polynomials of degree $\leqq 2 n-2$ is equivalent to the equations

$$
c_{k}=A_{1} x_{1}{ }^{k}+A_{2} x_{2}^{k}+\cdots+A_{n} x_{n}{ }^{k} \quad(k=0,1, \cdots, 2 n-2) .
$$

This system of equations may be written as the following matrix equation:

$$
X_{n}{ }^{T} D_{n} X_{n}=C_{n}
$$

where $C_{n}$ is the symmetric moment matrix (2) and

$$
D_{n}=\left[\begin{array}{cccc}
A_{1} & 0 & \cdots & 0 \\
0 & A_{2} & \cdots & 0 \\
\vdots & \vdots & \vdots & \vdots \\
0 & 0 & \cdots & A_{n}
\end{array}\right] \quad X_{n}=\left[\begin{array}{ccccc}
1 & x_{1} & x_{1}{ }^{2} & \cdots & x_{1}{ }^{n-1} \\
1 & x_{2} & x_{2}{ }^{2} & \cdots & x_{2}{ }^{n-1} \\
\vdots & \vdots & \vdots & \vdots & \vdots \\
1 & x_{n} & x_{n}{ }^{2} & \cdots & x_{n}{ }^{n-1}
\end{array}\right]
$$

If $C_{n}$ is congruent to a diagonal matrix with $p$ positive and $q$ negative diagonal elements then there is a nonsingular $n \times n$ matrix $B$ for which $B^{T} C_{n} B$ is a diagonal matrix of the stated form. Since

$$
\left(X_{n} B\right)^{T} D_{n}\left(X_{n} B\right)=B^{T} C_{n} B
$$


then $D_{n}$ is also congruent to a diagonal matrix of the stated form. From the law of inertia ([1], p. 296-298) $D_{n}$ also has $p$ positive and $q$ negative elements and the proof is complete.

From this result Theorem 1 follows as a corollary since, if $w(x)$ is nonnegative, $C_{n}$ is positive definite and therefore congruent to a diagonal matrix with $n$ positive elements.

As a simple example consider a 2-point quadrature formula of the form

$$
\int_{-1}^{1}(3-5|x|) f(x) d x \simeq A_{1} f\left(x_{1}\right)+A_{2} f\left(x_{2}\right) .
$$

For this weight function the monomial integrals are $c_{0}=1, c_{1}=0, c_{2}=-1 / 2$, $c_{3}=0$. There are no real values of $x_{1}, x_{2}$ for which (3) can be made exact for $f(x)=1, x, x^{2}, x^{3}$. There are, however, an infinity of such formulas with real $x_{1}$, $x_{2}$ which are exact for $f(x)=1, x, x^{2}$ and Theorem 2 still applies. One such formula is

$$
\begin{aligned}
x_{1} & =\frac{1}{2} & x_{2} & =1 \\
A_{1} & =2 & A_{2} & =-1 .
\end{aligned}
$$

Computation Center

University of Kansas

Lawrence, Kansas

1. F. R. Gantmacher, Matrix Theory, Vol. 1, Chelsea, New York, 1959.

2. V. I. KRYLov, Approximate Calculation of Integrals, Macmillan, New York, 1962. (Translated from Russian).

3. G. Szego, Orthogonal Polynomials, Amer. Math. Soc. Colloquium Publ., v. 23, 1959.

\section{A Partition Problem}

\section{By M. H. McAndrew}

1. Introduction. The following theorem is proved: Given integers $a, b, c, d$, each $\geqq 2$, then either there exist integers $m, n$ with $|m-n| \leqq 1$, a partition of a into $m$ parts of which each part is coprime to $b$, and a partition of $c$ into $n$ parts, each part coprime to $d$; or the same conclusion holds with the roles of $a$ and $b$ reversed and the roles of $c$ and $d$ reversed.

This question arises in the investigation of the minimum length of input strings required to distinguish two partial automata. Elgot and Rutledge [1] deduce an upper bound for the length of such strings and by using the theorem quoted above show that this upper bound can be attained. In Section 4 we demonstrate by an example that the restriction " $a, b, c, d \geqq 2$ " cannot be relaxed.

2. Preliminary Lemmas. In the sequel, all variables are to be taken as strictly positive integers.

Lemma 1. If $l>1, l=\prod_{i=1}^{r} P_{i}$ where the $p_{i}$ are distinct primes, and if $m$ is even, then there is an a such that

$$
\begin{aligned}
(a, l) & =1, \\
(m-a, l) & =1 .
\end{aligned}
$$

Received November 16, 1962. 